\title{
Extracorporeal membrane oxygenation support for a multitrauma patient with ARDS: A case report and literature review
}

\author{
YUEXING TU ${ }^{1 *}$, QI JIN ${ }^{2 *}$, RENHUA SUN ${ }^{1}$ and QIAN LI ${ }^{1}$ \\ ${ }^{1}$ Department of Critical Care Medicine, Zhejiang Provincial People's Hospital, People's Hospital of \\ Hangzhou Medical College, Hangzhou, Zhejiang 310014; ${ }^{2}$ Department of Critical Care Medicine, \\ Zhejiang Provincial Tiantai County People's Hospital, Taizhou, Zhejiang 317200, P.R. China
}

Received April 2, 2017; Accepted October 6, 2017

DOI: $10.3892 /$ etm.2017.5604

\begin{abstract}
Extracorporeal membrane oxygenation (ECMO) provides rescue treatment for patients with severe acute respiratory distress syndrome (ARDS) when conventional management fails. The present study reported ECMO as a life-saving treatment for ARDS due to bilateral pulmonary contusion in a 24-year-old male multiple trauma patient. ECMO therapy lasted 7 days for the patient and was successively stopped without any complications. ECMO modality should be chosen according to the respiratory and circulatory condition of each individual. Venoarteria-ECMO may be chosen for multiple trauma patients with ARDS, and respiratory and circulatory failure. Morphological changes are observed with ARDS progression. The injured lungs may recover completely with effective ECMO therapy.
\end{abstract}

\section{Introduction}

Acute respiratory distress syndrome (ARDS) is a form of noncardiogenic pulmonary edema, due to alveolar injury secondary to an inflammatory process, that can be either pulmonary or non-pulmonary origin (1). It is reported that about $4.6 \%$ of adult trauma patients develop ARDS (2). When conventional treatments fail, extracorporeal membrane oxygenation (ECMO) may be the rescue therapy for the patient (3). Here we demonstrate the rescue use of ECMO in an ARDS patient with severe bilateral pulmonary contusions with multiple injuries.

Correspondence to: Dr Qian Li, Department of Critical Care Medicine, Zhejiang Provincial People's Hospital, People's Hospital of Hangzhou Medical College, 158 Shangtang Road, Hangzhou, Zhejiang 310014, P.R. China

E-mail: zjicudr@126.com

${ }^{*}$ Contributed equally

Key words: ECMO, ARDS, multiple trauma

\section{Case report}

A 24-year-old male was involved in a road traffic accident as the driver of a motorcycle. The motorcycle hit the street tree at 50 miles per hour. The man suffered blunt thoracic and right lower limb trauma and was transferred to the nearest hospital about $2 \mathrm{~h}$ after the accident. Emergency chest computerized tomography (CT) showed (Fig. 1): Severe bilateral pulmonary contusions and pneumothorax with right serial rib fractures. And he also had right femoral intertrochanteric fracture. Bilateral chest drainage and right lower limbs fixation and traction were performed. The patient presented continuous tachypnea and worsened hypoxemia, therefore, he was intubated and ventilated with $\mathrm{FiO}_{2}$ 60-80\% after admission into ward. He was transferred to our hospital on the 5th day after the trauma for further therapy.

On arrival, the patient had a mean arterial pressure of $140 / 70 \mathrm{mmHg}$ with heart rate of $90 \mathrm{~min}^{-1}$, a $\mathrm{SpO}_{2}$ of $95 \%$ on ventilation oxygen $80 \%$, and a temperature of $39^{\circ} \mathrm{C}$. The chest CT taken at the arrival day showed (Fig. 2): Severe bilateral pulmonary contusions with right pulmonary consolidation. Pulmonary function and circulation of the patient deteriorated progressively during the next $10 \mathrm{~h}$. He was ventilated with the maximal settings (pressure controlled ventilation at $\mathrm{FiO}_{2} 1$ and PEEP $20 \mathrm{mmHg}$ ), with poor oxygenation $\left(\mathrm{SpO}_{2} 55-70 \%\right.$, $\mathrm{PaO}_{2} / \mathrm{FiO}_{2}<40 \mathrm{mmHg}$ ), and hemodynamic instability (central venous pressure [CVP] $10 \mathrm{mmHg}$, blood pressure [BP] $80 / 50 \mathrm{mmHg}$ with norepinephrine infusion at $\left.0.3 \mu \mathrm{g} \mathrm{kg}^{-1} \mathrm{~min}^{-1}\right)$. Urine output was less than $0.5 \mathrm{ml} / \mathrm{kg} / \mathrm{h}$ for more than $12 \mathrm{~h}$. Transthoracic echocardiography showed enlarged left atrium and decreased left ventricular systolic function with ejection fraction as $42 \%$. Epinephrine was subsequently infused at $0.18 \mu \mathrm{g} \mathrm{kg}^{-1} \mathrm{~min}^{-1}$ due to the low EF.

Considering the refractory hypoxia and shock, the patient was diagnosed as refractory ARDS, and that ECMO was the only remaining option. Left femoral artery (return) and left femoral vein (drainage) were cannulated under local anesthesia to establish venoarterial (VA)-ECMO with a flow of 4.5 litre $\mathrm{min}^{-1}$ and gas-blood ratio 1:1. The ECMO circuit consisted of a Maquet Rotaflow centrifugal pump (Maquet Cardiovascular, Rastatt, Germany), a BE-PLS2050 oxygenator 
(Maquet Cardiovascular). Continuous vein-vein hemofiltration $(\mathrm{CVVH})$ was used for oliguria and pulmonory edma by combination hemofiltration device with ECMO through connecting the inflow line of the CVVH circuit to the venous limb of ECMO circuit prior to the centrifugal pump, and the outflow line of the CVVH to the venous line of ECMO before the oxygenator (Fig. 3). Anticoagulation for ECMO and CVVH circuit was performed according to our institutional ECMO protocol with an initial bolus of heparin $100 \mathrm{IU} / \mathrm{kg}$ at the time of ECMO initiation followed by a continuous infusion of intravenous heparin, targeting an activated partial thromboplastin time (aPTT) between 60 and $80 \mathrm{sec}$. During ECMO, the mechanical ventilation was set down with a mean airway pressure of $20 \mathrm{mmHg}$ and $\mathrm{FiO}_{2}$ of 0.4 to prevent further barotrauma and oxygen toxicity.

The patient was successfully weaned from ECMO after $168 \mathrm{~h}$ and the femoral cannulas were removed. The daily ECMO setting parameter and ventilaor and hemodynamic parameters were recorded (Tables I and II). CT scan 2 days after weaning showed that bilateral consolidation of lungs became attenuated but still notable (Fig. 4). During ECMO therapy, there was no evidence of ECMO related bleeding and infection. The CVVH was weaned after 15 days. During the hospitalization, the patient underwent a tracheostomy and remained intubated for 3 weeks thereafter. He also had an open reduction and internal fixation for right intertrochanteric fracture. He was discharged from our hospital 43 days after his admission. CT scan 2 months after discharge showed that his lungs were almost completely recovered (Fig. 5).

\section{Discussion}

Many trauma patients with severe, bilateral pulmonary contusions may be managed by conventional therapies, but some of them can't survive without the rescue treatments of ECMO. This patient was injured mainly on the chest initially and he remained acceptable oxygenation although supported by a relatively high mechanical ventilation. However, his respiratory and circulation deteriorated in the following week. The sever ARDS and shock might be the consequence of inflammation cascade of the trauma hit, and the development of pulmonary infection or ventilator-induced lung injury. It can be differentiated from other cause of respiratory failure and cardiac shock such as pulmonary embolism, acute coronary syndrome based on his trauma history, progessing of clinical manifestation and examination. Published experience suggests that ECMO could be an option for salvage therapy to trauma patient with sever pulmonary failure like this case (4). According to the Extracorporeal Life Support Organization (ELSO; https://www.elso.org/Resources/Guidelines.aspx). ECMO initiation should be considered in hypoxic respiratory failure when the risk of mortality is $50 \%$ or greater, identified by $\mathrm{PaO}_{2} / \mathrm{FiO}_{2}$ less than $150 \mathrm{mmHg}$ on $\mathrm{FiO}_{2}$ more than $90 \%$ and/or Murray score 2-3, and is indicated when this risk exceeds $80 \%$, that is, when $\mathrm{PaO}_{2} / \mathrm{FiO}_{2}$ is less than $80 \mathrm{mmHg}$ on $\mathrm{FiO}_{2}$ more than $90 \%$ and Murray score is 3-4 or hypercapnia with a $\mathrm{PaCO}_{2}$ more than $80 \mathrm{mmHg}$ or inability to achieve safe inflation pressures (Pplat $30 \mathrm{~cm} \mathrm{H}_{2} \mathrm{O}$ ). And this case had strong indication to initiate ECMO therapy and proved to be successfully recovered finally.

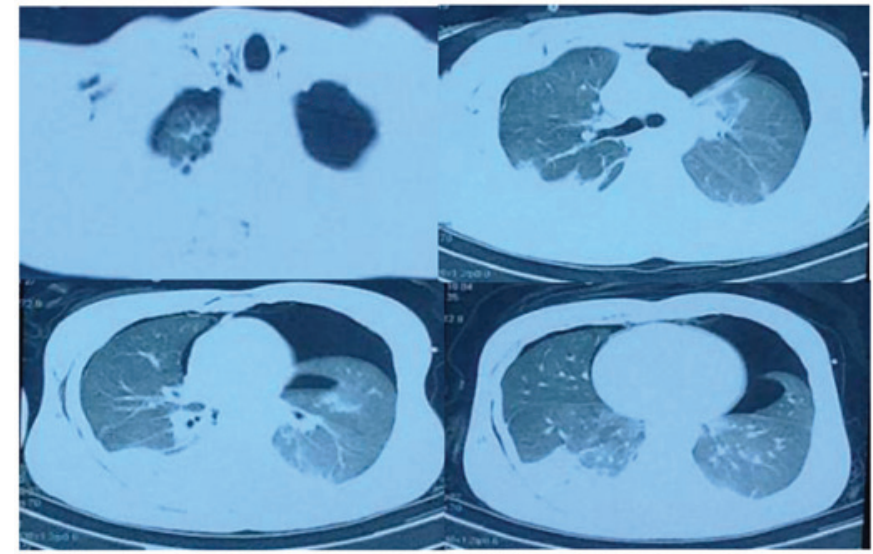

Figure 1. Chest CT scan images (lung window) at the 1st day of trauma showed bilateral ground glass infiltrate and pneumothorax.

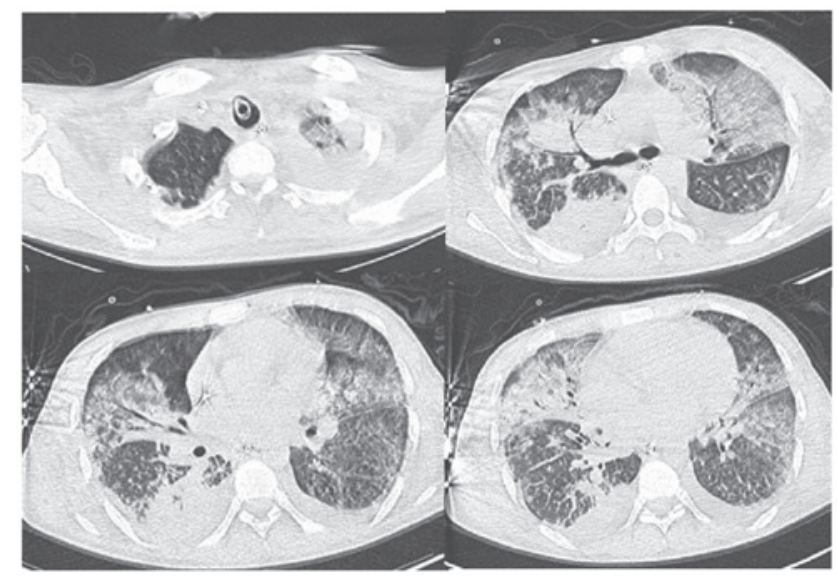

Figure 2. Chest CT scan images (lung window) at the day before ECMO showed bilateral consolidation.

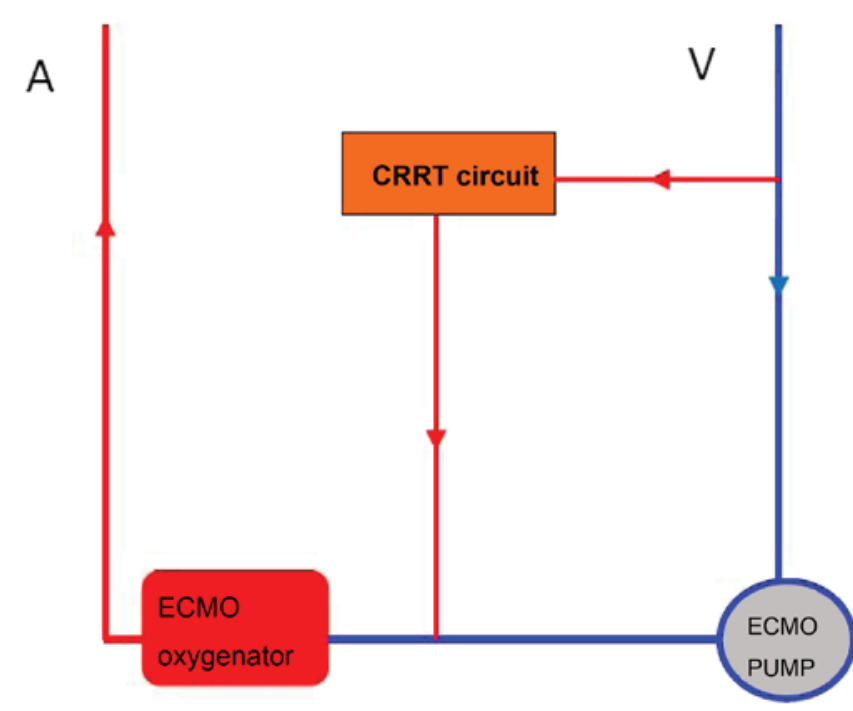

Figure 3. Combining ECMO and CRRT circuit. A: access back to artery; $\mathrm{V}$ : access from vein; CRRT, continuous renal replacement therapy; ECMO, extracorporeal membrane oxygenation.

There are primarily two types of ECMO according to the route of access: Venovenous(VV)-ECMO and venoarterial 
Table I. Daily ECMO setting parameters.

ECMO parameter

\begin{tabular}{lcc}
\cline { 2 - 3 } Day & Blood flow (1/min) & Gas flow (1/min) \\
\hline 1st & 4.5 & 2.25 \\
2nd & 4.5 & 2.25 \\
3rd & 4 & 2 \\
4th & 4 & 2 \\
5th & 3 & 1.5 \\
6th & 2.4 & 1.2 \\
7th & 2 & 1 \\
\hline
\end{tabular}

Table II. Ventilaor and hemodynamic parameters on the ECMO weaning day.

\begin{tabular}{lc}
\hline Mode & Pressure control \\
\hline Ventilator & 35 \\
$\mathrm{FiO}_{2}(\%)$ & 12 \\
Frequecy $(\mathrm{bpm})$ & 20 \\
Mean pressure $(\mathrm{ml})$ & 25 \\
Peak pressure $\left(\mathrm{cmH}_{2} \mathrm{O}\right)$ & 5 \\
Positive end-exrespiratory pressure & \\
(cmH $\left.\mathrm{C}_{2} \mathrm{O}\right)$ & \\
Hemodynamics & 75 \\
Mean arterial pressure $\left(\mathrm{cmH}_{2} \mathrm{O}\right)$ & 12 \\
Central venous pressure $\left(\mathrm{cmH}_{2} \mathrm{O}\right)$ & 82 \\
Heart rate $(b p m)$ & \\
\hline
\end{tabular}

(VA)-ECMO (5). In the retrospective review (6), the proportion of initial VV-ECMO support for adult ARDS patients has increased to $86 \%$. VV-ECMO has become the first-choosen modality for simple respiratory failure in adults considering its ability to effectively oxygenate and ventilate patients with lower risks. VA is preferred if cardiac function is moderately to severely depressed and cardiac support is also required. Although VA-ECMO are reported with increased risk of stroke (7), bleeding (7), renal failure (8), and Harlequin syndrome (9) (selective lower body oxygenation with upper body hypoxia), VA-ECMO can offer hemodynamic support for a cardiogenic cause of hypotension. For this case, cardiac shock was confirmed by clinical examination, echocardiogram. Therefore, we used VA-ECMO considering the unstable blood pressure and decreased heart function. VA-ECMO not only provides oxygen saturation and delivery that permits reduced ventilator settings and 'lung rest', but also relieves the restricted and congested heart by unloading the right atrium and ventricle. It is very important to monitor the right upper body saturation, the coronary and brain perfusion during VA-ECMO, particularly while left ventricular dysfunction improving and pulmonary function deteriorating (10).

In this case, continuous renal replacement therapy (CRRT) was used for oliguria and pulmonory edma

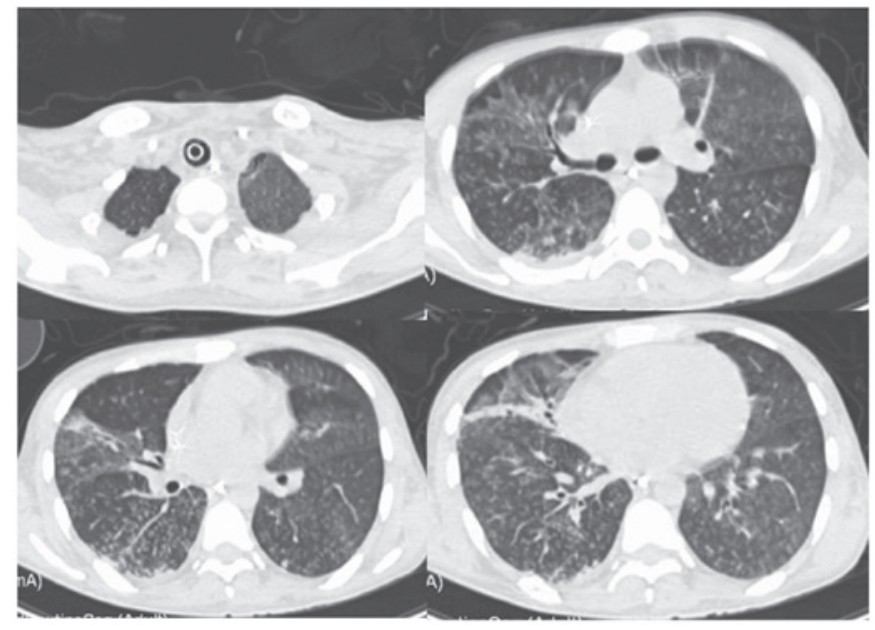

Figure 4. Chest CT scan images (lung window) 2 day after ECMO weaning showed attenuated consolidation.

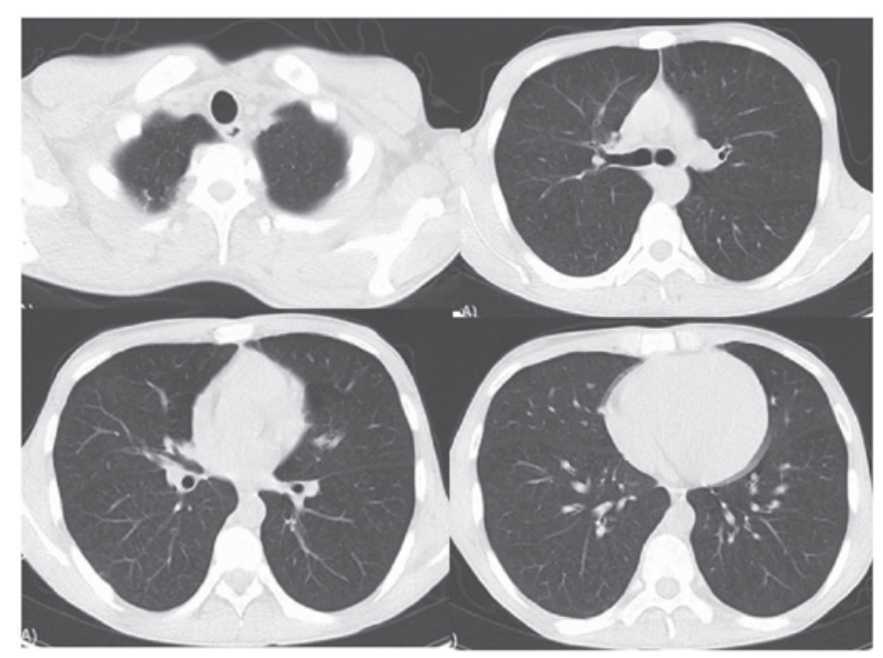

Figure 5. Chest CT scan images (lung window) 2 months after discharge showed recoverd lungs.

combined with ECMO. It has been reported that fluid overload during ECMO support is associated with increased risk of mortality (11). CRRT is an important and efficient therapy that can be combined with ECMO to treat fluid overload and AKI. Fluid restriction has been confirmed to have an overall beneficial effect on ARDS-associated morbidity (12). CRRT provides an easily initiated and efficient method of fluid management. We used CRRT for 15 days until the kidney could work normally.

In this case, we took several CT checks for the patient during the hospitalization and after discharge. The lungs demonstrated typical ground-glass infiltrate, dense consolidation changes in turn and did not recovered even till the weaning of ECMO. However, the CT presented with only little residual shadows of fiber ropes 2 months after discharge. Pulmonary parenchymal damages during ARDS progression determined degree of pulmonary fibrosis that can be detected by imaging checks, and thus ARDS patients' prognoses differ significantly according to the morphological changes (13). A follow-up investigation shows that $\mathrm{CT}$ scanning results of patients after 
ECMO shows various degrees of morphological changes in the lungs and the severity of these changes may be associated with the disease duration (14). ECMO lasted 7 days in this case and the finally almost completely recovered lung images indicated little injury occured during the course. ECMO may be helpful to reduce the lungs injury and prompt their recovery.

ECMO can be used as a rescue therapy in multi-trauma patient with severe ARDS. VA-ECMO modality should be chosen very discreetly considering the respiratory and circulatory function of the ARDS patient. ECMO is beneficial to relieve the injury to the ARDS lungs.

\section{References}

1. Sweeney RM and McAuley DF: Acute respiratory distress syndrome. Lancet 388: 2416-2430, 2016.

2. Chaiwat O, Lang JD, Vavilala MS, Wang J, MacKenzie EJ, Jurkovich GJ and Rivara FP: Early packed red blood cell transfusion and acute respiratory distress syndrome after trauma. Anesthesiology 110: 351-360, 2009.

3. Huang YK, Liu KS, Lu MS, Wu MY, Tsai FC and Lin PJ: Extracorporeal life support in post-traumatic respiratory distress patients. Resuscitation 80: 535-539, 2009.

4. Madershahian N, Wittwer T, Strauch J, Franke UF, Wipperman J, Kaluza M and Wahlers T: Application of ECMO in multitrauma patients with ARDS as rescue therapy. J Card Surg 22: 180-184, 2007.

5. Haft $\mathrm{J}$ and Bartlett R: Extracorporeal membrane oxygenation (ECMO) in adults. UpToDate [Internet]. Version 15.0. Waltham (MA): UpToDate; c2005, 2013.

6. Kon ZN, Bittle GJ, Pasrija C, Pham SM, Mazzeffi MA, Herr DL, Sanchez PG and Griffith BP: Venovenous versus venoarterial extracorporeal membrane oxygenation for adult patients with acute respiratory distress syndrome requiring precannulation hemodynamic support: A review of the ELSO registry. Ann Thorac Surg 104: 645-649, 2017.
7. Delius R, Anderson H III, Schumacher R, Shapiro M, Otsu T, Toft K, Hirsch J and Bartlett R: Venovenous compares favorably with venoarterial access for extracorporeal membrane oxygenation in neonatal respiratory failure. J Thorac Cardiovasc Surg 106: 329-338, 1993.

8. Oshima K, Kunimoto F, Hinohara H, Ohkawa M, Mita N, Tajima Y and Saito S: Extracorporeal membrane oxygenation for respiratory failure: Comparison of venovenous versus venoarterial bypass. Surg Today 40: 216-222, 2010.

9. Papademetriou MD, Tachtsidis I, Banaji M, Elliott MJ, Hoskote A and Elwell CE: Optical topography to measure variations in regional cerebral oxygenation in an infant supportedon veno-arterial extra-corporeal membrane oxygenation. Adv Exp Med Biol 737: 71-76, 2012.

10. Shekar K, Mullany DV, Thomson B, Ziegenfuss M, Platts DG and Fraser JF: Extracorporeal life support devices and strategies for management of acute cardiorespiratory failure in adult patients: A comprehensive review. Crit Care 18: 219, 2014

11. Selewski DT, Cornell TT, Blatt NB, Han YY, Mottes T, Kommareddi M, Gaies MG, Annich GM, Kershaw DB, Shanley TP and Heung M: Fluid overload and fluid removal in pediatric patients on extracorporeal membrane oxygenation requiring continuous renal replacement therapy. Crit Care Med 40: 2694-2699, 2012.

12. National Heart, Lung, and Blood Institute Acute Respiratory Distress Syndrome (ARDS) Clinical Trials Network, Wiedemann HP, Wheeler AP, Bernard GR, Thompson BT, Hayden D, deBoisblanc B, Connors AF Jr, Hite RD and Harabin AL: Comparison of two fluid management strategies in acute lung injury. N Engl J Med 354: 2564-2575, 2006.

13. Masclans JR, Roca O, Muñoz X, Pallisa E, Torres F, Rello J and Morell F: Quality of life, pulmonary function, and tomographic scan abnormalities after ARDS. Chest 139: 1340-1346, 2011.

14. Li XY, Sun B, Wang CT, He XY, Zhang CY, Ding Y, Peng P, Tong ZH and Zhan QY: A follow-up study on acute respiratory distress syndrome survivors after extracorporeal membrane oxygenation by pulmonary high-resolution CT. Arch Iran Med 18: 6-11, 2015. 